\title{
Index autorum ad Vol. 149
}

\section{Confecit F. Ammann, Geneve.}

(B) $=$ Buchbesprechungen - Livres nouveaux - Book reviews. (V) $=$ Vortrag - Communication Report.

AichmaiГ, H., 463 Amsler, M., 438 (V) Azevedo, M. L. de, v. De Azevedo, M. L.

Babel, J., 277 (V)

Balen, A. Th. M. van, v. Van Balen,

A. Th. M. Berg, E. O. van den, v. Van den

Berg, E. O. Bodenheimer, A. R., 424 (V) Bokor, S., 86

Boniuk, M. (Editor), 505 (B) Bruckner, A., 504 (B) Bruckner, R., 497 Bucklers, M., 37 Butler, I., 204 (V)

Colenbrander, M. C, Ill (V),

$142(\mathrm{~V}), 146(\mathrm{~V})$

D’Alessandro, C, 161 De Azevedo, M. L., 43 De Jorge, F. B., 43

Eröss, S., 27

Franceschetti, A., 266 (V) , 344 (V) Francois, J., 53 Fulmek, R., 484

Gailloud, CL, 505 (B)

Gobin, M. H., 138 (V), 146 (V)

Goldmann, H., 481

Hamburg, A., 21, 151 (V) Hanisch, J., 27 Hanssens, M., 53

Henkes, H. E., 202 (V), 208 (V),

223 (V), 225 (V) Hilsdorf, C, 12

Houtsmuller, A. J., 208 (V), 246 (V) Huber, A., 359 (V)

Jorge, F. B. de, v. De Jorge, F. B. Jütte, A., 3

Kálló, A., 27

Klöti, R., 152

Koten, J. W., 151 (V)

Kurstjens, J. H., 221 (V)

Lagasse, A., 53 Lammens, A. J. J., 210 (V) Landolt, E., 405 (V) Lang, J., 456 Lawitschka, F., 484 Lemke, L., 3 Lund, O.-E., 248 (B)

Maione, M., 81 Malbran, E., 161 Mammarella, E., 81 Manschot, W. A., 121 (V), 131 (V), 241

(V)

Oksala, A., 467 Oomen, H. A. P. C, 197 (V) Oosterhuis, J. A., 210 (V) Orbán, T., 27

Patakfalvi, A., 86 Paul, S. D., 177

514

Index autorum ad Vol. 149

Renard, G. (Editor), 504 (B) Rintelen, F., 68, 312 (V) Rivara, A., 185 Rohen, J. W., 505 (B)

Rosselet, E., 416 (V)

Schmidt, Th., 250 (V), 481 
Schweitzer, N. M. J., 230 (V)

Sebestyén, J., 86

Seitz, R., 504 (B)

Senus, A. H. C. van, v. Van Senus,

A. H. C. Speiser, P., 447 Stelzer, R., 96

Streiff, E. B. (Editor), 504 (B) Streiff, E. B., 248 (B),504 (B)

Troelstra, A., 230 (V)

Valenzuela, J., 161 Van Balen, A. Th. M., 246 (V) Van den Berg, E. O., 236 (V) Van Senus, A. H. C, 228 (V) Verrey, F., 375 (V)

Weber, G., $326(\mathrm{~V})$

Witmer, R., 152, 295 (V), 390 (V),

4. 447 Worst

J. G. F.

$118(\mathrm{~V})$

$227(\mathrm{~V})$

Zagora

E.

504 (B) Zingirian

M.

185 